\title{
Highly Regular Polyampholytic Structures Adsorbed Directly from Solution
}

\author{
Mahltig B(a), Müller-Buschbaum $\mathrm{P}^{(\mathrm{b})}$, Wolkenhauer $\mathrm{M}^{(\mathrm{c})}$ Wunnicke $\mathrm{O}^{(\mathrm{a})}$, Wiegand $\mathrm{S}^{(\mathrm{b})}$ Gohy J- \\ $\mathrm{R}^{(\mathrm{d})}$, Jérôme $\mathrm{R}^{(\mathrm{d})}$ and $\operatorname{Stamm} \mathrm{M}^{(\mathrm{a})}$ \\ (a) Institut für Polymerforschung Dresden eV, Hohe Str 6, D-01005 Dresden, Germany, \\ (b)Physik-DepartmentLS E13, Technische Universität München, James-Pr anck-Str. 1, D-85747 \\ Garching, Germany, \\ (c) Max-Planck-Institut für Polymerforschung, Ackermannweg 10, D-55I28 Mainz, Germany, \\ and \\ (d) Centre for Education and Research on Macromolecules, University of Liège, Sart-Tilman B6, B- \\ 4000 Liège, Belgium
}

\begin{abstract}
This article concerns the adsorption of the diblock polyampholyte poly(methacrylic acid)-blockpoly((dimethylamino)ethyl methary-late) (PMAA-b-PDMAEMA) from aqueous solution on silicon substrates. The investigated polyampholyte is characterized by a small molecular weight around 15,000 $\mathrm{g} / \mathrm{mol}$ and a big positively charged PDMAEMA block. The adsorbed amount determined by ellipsometry was str ongly influenced by the $\mathrm{pH}$ of the adsorption solution. Using dynamic light scattering polyampholytic structures with diameters around $50 \mathrm{~nm}$ were found in aqueous solution. The hydrodynamic diameter was hardly affected by changing the $\mathrm{pH}$ of the polymer solution. Analogous regular structures were also found by scanning force microscopy (SFM) and grazing incidence, small angle X-ray scattering (GISAXS) at the silicon surface after the adsorption process. While SFM provides a topographical image of a small part of the adsorbed polyampholytic layer; GISAXS was used to get a statistical description of the lateral surface structures. The adsorbed structures were highly regular and their sizes were nearly $\mathrm{pH}$ independent over a lar ge $\mathrm{pH}$ region. Only directly at the isoelectric point of the polyampholyte larger adsorbed structures were observed. Compared with earlier investigated PMAA-b-PDMAEMA systems we are now able to prepare highly regular polyampholytic structures at silicon surfaces. There are two kinds of interactions for the adsorbed micelles. First, the charged block of the chains is directly attracted to the substrate via electrostatic interactions, while the uncharged part of the chains is only hydrophobically attracted via the hydrophobic core of the adsorbed micelle.
\end{abstract}

Key Words: polyampholyte; adsorption; desorption; micelles; SFM; GISAXS; ellipsometry.

\section{Introduction}

Polyelectrolytes containing a high amount of oppositely charged functional groups are called polyampholytes [1]. These types of charged macromolecules are widely used in many technical applications such as paper production, wastewater treatment, and washing powder [2-5]. In most of these applications the polyelectrolyte affected surface abilities via grafting or adsorption at the solid/liquid interface. Therefore there is much scientific interest in the adsorption behavior of polyelectrolytes and polyampholytes.

Polyelectrolytes in solution and in adsorption processes are well described experimentally and theoretically in the literature [6-10]. Single polyampholytic chains in solution were theoretically described by Higgs and Joanny [11]. In solution also the formation of multichain aggregates or micelles were found experimentally $[12,13]$ and were expected to have a strong influence on the adsorption process from these solutions $[14,15]$. The adsorption of statistical and diblock polyampholytes was reported by many authors [16-21] Therefore our investigations not only concern the single adsorption process. The aim of this article is to report the creation of highly regular polyampholytic structures at the solid/liquid interface adsorbed directly from solution. Another view will also concern the desorption behavior of these polyampholytic layers and will give a further insight in the structure of the polyampholyte layer.

These investigations were performed with the new diblock polyampholyte poly(methacrylic acid)block;-poly((dimethyl- amino)ethyl methacrylate) (PMAA- $b$-PDMAEMA). Until now there have been 
some articles concerning the solution behavior of this polyampholyte [12,13]. Many parameters of the aqueous polyampholyte solution such as the $\mathrm{pH}$, the salt, and the polymer concentration have significant influence on the adsorption behavior [20,21]. Also the influence of the poly ampholyte characteristics, molecular weight, and block ratio has been reported [22] Depending on the adsorption conditions different kinds of adsorption phenomena were observed. Besides single chain adsorption the adsorption of whole micelles on silicon substrates was observed also [14, 15, 22].

In the present paper, the adsorption was performed on silicon substrates as a function of the $\mathrm{pH}$ of the aqueous poly ampholyte solution. The adsorbed amount was determined using ellipso-metry, while the lateral structures of adsorbed polyampholyte, were investigated by scanning force microscopy (SFM) and by grazing incidence, small angle X-ray scattering (GISAXS). The found lateral sizes were compared with the hydrodynamic diameters of polyampholytic structures observed in aqueous solutions using dynamic light scattering (DLS)

\section{EXPERIMENT}

\subsection{Polymer and Substrate}

The adsorption experiments were performed with the di-block polyampholyte PMAA- $b$-PDMAEMA. This polyam-pholyte was synthesized using anionic polymerization, while the molecular weight and the block ratio were determined by gel permeation chromatography and nuclear magnetic resonance spectroscopy $\left({ }^{1}\right.$ H-NMR), respectively [23-25]. The molecular weight of the used polyampholyte was determined to be $\mathrm{M}_{\mathrm{n}}=15000 \mathrm{~g} / \mathrm{mol}$, while the weight ratio of the two blocks PMAA: PDMAEMA was $33: 67$. The isoelectric point (IEP) of the polyampholyte determined using electrokinetic measurements was $\mathrm{pH}_{\text {IEP }}=8.5$ [15] As substrates silicon wafers with a siliconoxide layer of approximately $2 \mathrm{~nm}$ were used All substrates were cleaned using an alkalic cleaning procedure with an aqueous mixture of $\mathrm{NH}_{3}$ and $\mathrm{H}_{2} \mathrm{O}_{2}$ at $70^{\circ} \mathrm{C}$. After the wafers were rinsed intensively with Milli-Pore water and dried with nitrogen, the IEP of the silicon substrates determined by streaming potential measurements was $\mathrm{pH}_{\text {IEP }}$ $=3.8[15]$.

\subsection{Sample Preparation}

All adsorption experiments were carried out from aqueous solutions with different $\mathrm{pH}$ values. These solutions contained a polyampholyte concentration of $013 \mathrm{~g} / \mathrm{L}$ Additional $\mathrm{NaCl}$ or $\mathrm{CsCl}$ were added in a concentration of $0.01 \mathrm{~mol} / \mathrm{l}$. The $\mathrm{CsCl}$ was added in case of the sample preparation for GISAXS experiments to increase the sensitivity of the adsorbed polyam-pholyte layer in such X-ray scattering experiments. The $\mathrm{pH}$ of the polyampholytic solution was set by adding acid $(\mathrm{HCl})$ or base $(\mathrm{NaOH})$ in small amounts. After the preparation of the adsorption solution the silicon substrate was placed in this solution for at least $10 \mathrm{~h}$. When the sample was taken out of the adsorption solution, it was rinsed with Milli-Pore water. After drying, the adsorbed polyampholytic layer was investigated using ellipsometry, SFM, and GISAXS.

The desorption of the polyampholytic layer was carried out with THF In this case silicon substrates carrying an adsorbed polyampholyte layer were placed into pure THF After at least $2 \mathrm{~h}$ the substrates were taken out of the solution, rinsed with Milli-Pore water and dried with nitrogen,

To check the stability of the adsorbed polyampholyte film as function of temperature the films were annealed up to $150^{\circ} \mathrm{C}$ under vacuum. After at least $6 \mathrm{~h}$ annealing, the samples were cooled down to room temperature and their topographies were investigated with SFM

\section{Ellipsometry}

The adsorbed amount of polyampholyte was determined using a computer controlled null ellipsometer in a polarizer-compensator-sample-analyzer arrangement (Multiskop, Optrel Berlin) [26], As light source a $A$ He-Ne laser with $\lambda=632.8 \mathrm{~nm}$ was used and the angle of incident was set to $70^{\circ}$. A multilayer model for homogeneous films covering the silicon wafers were used to calculate the layer thickness $d$ of the adsorbed polyam-pholyte layer from the ellipsometric angles $\psi$ and $\Delta$ [27]. The adsorbed amount $A$ was determined from the layer thickness $d$ measured in air after the adsorption process by $A=\delta d$. With $\delta=1.27 \mathrm{~kg} / 1$ as the mass density of the adsorbed polymer layer. 


\section{Dynamic Light Scattering}

To determine the dimensions of polyampholytic structures in solution, dynamic light scattering measurements were performed The device was built up from a commercial ALV 3000 digital correlator A $400-\mathrm{mW}$ krypton laser $(\lambda=300 \mathrm{~nm})$ was used as a light source The analysis of the autocorrelation function lead to the diffusion coefficient of the solved polymer structures. The size of these structures in solution was calculated from the related diffusion coefficient using the Stokes-Einstein equation $[28]$.

\subsection{Scanning Force Microscopy}

To study the topography of dried adsorbed polyampholytic layers the scanning force microscopy was used. All investigations were performed with a commercially available SFM (Multimode Nanoscope III/Digital Instruments) at air. The topographies were taken in the tapping mode to reduce any damage of the polyampholytic layer caused by tip contact. The measurement frequencies were set between 280 to $330 \mathrm{kHz}$. The used cantilevers were made from microfabricated silicon (Nanosen-sors).

Measurements at several sample positions were performed with scan areas between $1 \mathrm{x} 1 \mu \mathrm{m}$ and $20 \mathrm{x}$ $20 \mu \mathrm{m}$, To get a less pictoral view on the surface, the topographies observed by SFM were analyzed by a Fourier transformation [29] A better signal-to-noise ratio was reached in an additional next step with the calculation of the radially averaged power spectral density (PSD) from the Fourier transform: PSD $=1 / 2 \pi|\mathrm{F}(\mathrm{q})|^{2}$.If the PSD spectrum contained a peak, the peak position $s^{*}$ was extracted using a fit of a Gaussian line shape to the PSD [30]. From this position $s^{*}$ the dominant lateral length scale $S^{*}$ in the surface topography was then calculated:

$$
S^{*}=1 / s^{*}=2 \pi / q
$$

If a PSD spectrum of a SFM topography contained a shoulder instead of an exhibited maximum, the position $s^{*}$ would be determined using two linear fits. The fits were carried out on the left and the right side of the shoulder. The crossing point of both linear fits determined the position $s^{*}$.

To get additional information about the lateral structures of the adsorbed polyampholytic layers the grazing incidence small angle X-ray scattering was performed. This scattering technique supplements the force microscopy because GISAXS delivers information about lateral structures all over the illuminated sample area of some $\mathrm{mm}^{2}$, while the detection area of SFM is only limited to the range of several $\mu \mathrm{m}^{2}$.

The dried layers adsorbed from aqueous polyampholyte solutions containing $\mathrm{CsCl}$ were measured at the USAX beam-line BW4 at the DORIS III storage ring at HASYLAB/DESY in-Hamburg (Germany) $[31,32]$ The replacement of $\mathrm{NaCl}$ by $\mathrm{CsCl}$ in the adsorption solution causes an increased sensitivity of the adsorbed polyampholyte layer to the GISAXS experiments. The response of the adsorbed polymer films increased due to the bigger election density of the coadsorbed ions $\mathrm{Cs}^{+}$compared with $\mathrm{Na}^{+}$. The X-ray source monochromatisized set to a wavelength of $0.138 \mathrm{~nm}$ and the distance between sample and detector was set to $12.84 \mathrm{~m}$ The scattered intensity was recorded with a two-dimensional detector providing a resolution of the parallel in-plane transfer $q_{y}$ better than $0.002 \mathrm{~nm}^{-1}$. In this arrangement the xy-plane corresponds to the sample surface All measurements were performed in a reflection geometry to realize diffuse X-ray scattering in an ultra small angle arrangement. The two-dimensional intensity distribution separated in several vertical and horizontal slices was detected at a fixed angle of incidence $\alpha_{i}$. On the one hand the vertical slices [33] correspond to detector scans, which are mainly dependent on $q_{z}$ and include depth sensitive information [34] On the other hand the horizontal slices corresponding to so-called out-of-plane scans include in-plane information of the most prominent lateral size from $q_{y}$ dependence. The horizontal slices were taken at the angle $\alpha=\alpha_{i}+\alpha_{c}$, where $\alpha_{c}$ is the critical angle of total reflection for the polyampholyte At this position the horizontal slices include the contribution of lateral sizes in the adsorbed polyampholyte layer.

All shown out-of-plane scans are horizontal slices at this angle The most prominent, lateral length scale was determined from these out-of-plane scans using a fit of a Gaussian line shape.

\section{RESULTS AND DISCUSSION}

The amount of adsorbed polyampholyte was determined as function of $\mathrm{pH}$ of the adsorption solution (Fig. 1). From pH 2.9 to 8.5 an increase in the adsorbed amount from 9.8 to $27.3 \mathrm{mg} / \mathrm{m}^{2}$ was observed. 
At higher $\mathrm{pH}$ values the adsorbed amount decreased again and at $\mathrm{pH} 11.2$ no adsorption was determinable. The maximum in adsorption is near the electrokinetically determined IEP of the poly ampholyte with $\mathrm{pH}_{\mathrm{IEP}}$ 8.5. In this $\mathrm{pH}$ area around the IEP also a polyampholyte precipitation appeared, which was measured as a reduction of transmission of a red laser light passing the polyampholyte solution. Such a behavior is quite typical to polyampholytes at the IEP. Here the oppositely charged polymer parts carry the same amount of charged groups, so the attractive electrostatic interactions increased and favored polyampholytic precipitation $[1,11,35]$.

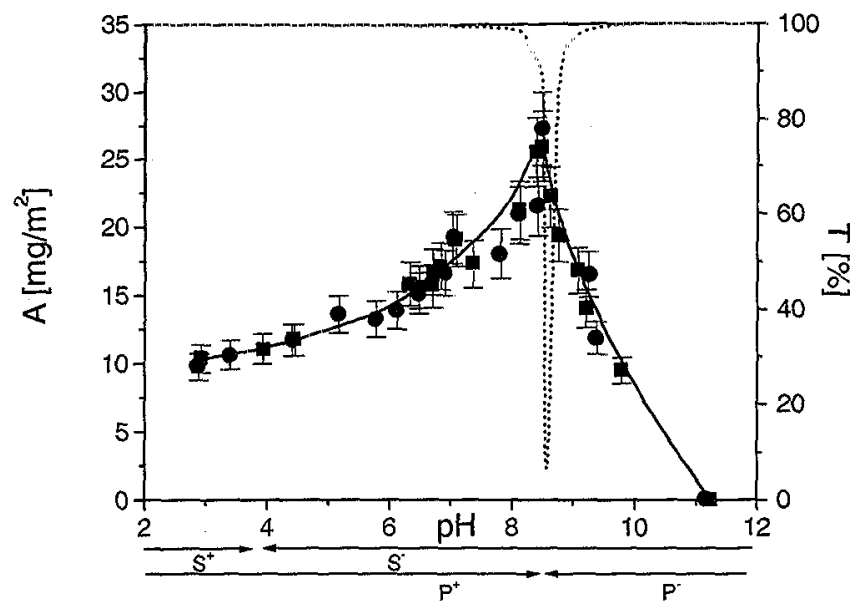

FIG. 1. Adsorbed polyampholyte amount $A$ as a function of $p H$ of the adsorption solution The adsorption was pet formed under the presence of $0.01 \mathrm{~mol} / 1 \mathrm{NaCl}$ (ロ) or $0.01 \mathrm{~mol} / 1 \mathrm{CsCl}(\bullet)$. The solid line is shown as a guide for the eye and the dotted line presented the transmission T of a red laser light through the polyampholytic solution The arrows below mark the $\mathrm{pH}$ areas where the substrate $S$ and the polyampholyte $P$ are carrying a positive or negative net charge

The appearance of the adsorption maximum at the polyam-pholyt $\mathrm{pH}_{\text {IEP }} 8.5$ is also explainable by electrostatic interactions Both the silicon substrate and the polyampholyte are carrying electrostatic charges The oppositely charged polyampholyte block is expected to adsorb at the siliconoxide covering the silicon wafer, while the other polymerblock is placed away from the substrate $[17,36]$. In this scenario the amount of adsorbed poly ampholyte is limited by the net charge of the poly ampholyte In $\mathrm{pH}$ areas far away from the IEP the polyampholyte carrying a high net charge and between the adsorbing polymer chains repulsive electrostatic interactions limited the adsorption. Toward the IEP the net charge decreased and the electrostatic repulsion decreased also. So an adsorption in a higher density is favorable and the adsorbed amount increased toward the IEP [37].

The adsorption experiments were performed under presence of $0.01 \mathrm{~mol} / 1 \mathrm{NaCl}$ or $001 \mathrm{~mol} / 1 \mathrm{CsCl}$. Because of the higher electron density of $\mathrm{Cs}^{+}$compared with $\mathrm{Na}^{+}$, the $\mathrm{CsCl}$ was used to increase the response of the adsorbed polyampholyte films for-X-ray scattering experiments. In addition to the adsorption of polyampholyte chains also the solved ions attracted to these chains adsorbed and are present in the adsorbed polyampholytic structures at the surface $[9,10]$. If now salts with a higher electron density like $\mathrm{CsCl}$ instead of $\mathrm{NaCl}$ are used, the electrondensity of the adsorbed polyampholytic structures increased and are better detectable by X-ray scattering.

The adsorbed amount as function of $\mathrm{pH}$ is here not influenced by changing the kind of salt from $\mathrm{NaCl}$ to $\mathrm{CsCl}$. During the adsorption process the solved low molecular weight ions shielded the repulsive electrostatic interactions between the polymer chains Because both $\mathrm{NaCl}$ and $\mathrm{CsCl}$ are consisting only of monovalent ions the ionic strength of a $0.01-\mathrm{mol} / 1$ solution of these salts is quite similar and the shielding effect between the polymer chains is comparable [9, 20]. 

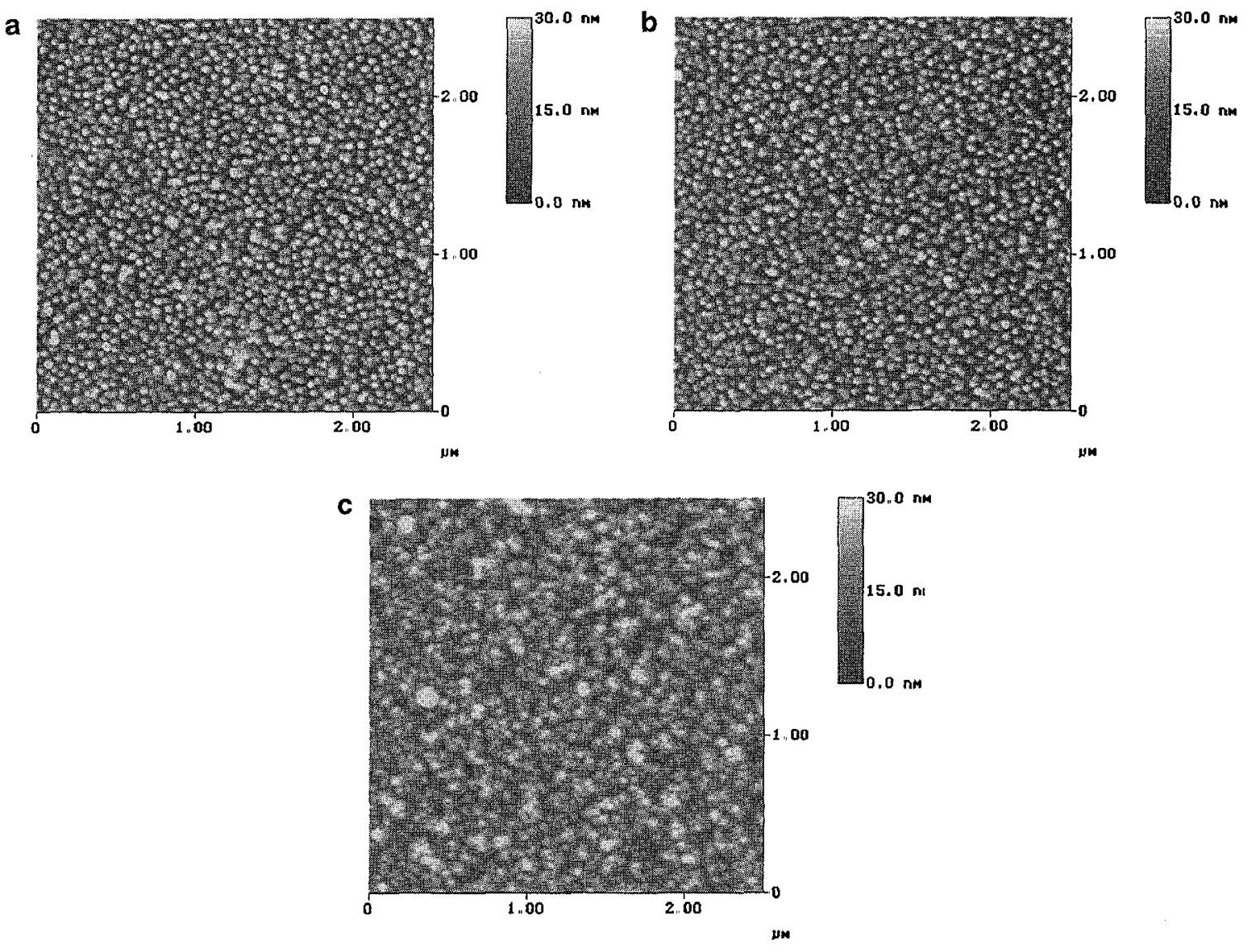

FIG. 2. SFM topographies of the polyampholyte adsorbed from solutions containing different $\mathrm{pH}$

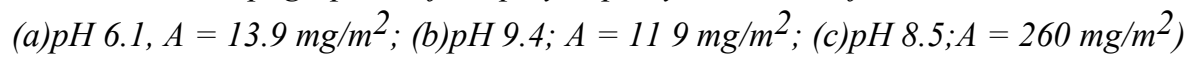

Therefore, the adsorbed amount is not affected by the kind of monovalent salt added The ellipsometrically determined adsorbed amount was a net value and included no additional information about the structure of the adsorbed polyampholyte on the substrate. The structural investigations were performed with SFM and GISAXS Figure 2 shows three topographies of the polyampholyte adsorbed from solutions containing different $\mathrm{pH}$. The PSD spectra calculated from these topographies giving the most prominent, lateral length scale of the adsorbed polyampholyte are shown in Fig.. 3 together with the out-of-plane scans measured with GISAXS, The adsorption at $\mathrm{pH}$ 6,1 and at $\mathrm{pH} 9.4$ led to quite similar adsorbed amounts of around $13 \mathrm{mg} / \mathrm{m}^{2}$. Also the surface topographies consisting of regular round structures with a lateral diameter around $60 \mathrm{~nm}$ and a height up to $30 \mathrm{~nm}$ were similar, The lateral diameter of the adsorbed structures was identified in the PSD spectra of the according SFM topography as the most prominent lateral length scale. Similar length scales were estimated from the out-of-plane scans of the GISAXS measurements So the prominent length determined from a SFM image of some $\mu \mathrm{m}^{2}$ sample area is similar to the prominent length determined by GISAXS collecting data over a $\mathrm{mm}^{2}$-sized area. This fact gives the reason that the highly regular structures observed by SFM cover the whole sample in high regularity.

The SFM topography and the PSD spectrum of the polyam-pholyte film adsorbed at $\mathrm{pH} 8.5$ near the IEP show different surface structures. There are no longer well-separated structures of regular lateral sizes The structures are close together and grown into each other (Fig. 2c). An analogous picture is shown by the PSD, which included no significant maximum. Only a shoulder in the PSD resulted in a lateral length scale of $179 \mathrm{~nm}$, which is much larger than in the case of samples at other $\mathrm{pH}$ values (Fig. 3). To explain now the different structures near the IEP of the polyampholyte and the regular-sized one formed at other $\mathrm{pH}$ areas the dominant length scales at the surface and the hydro-dynamic diameter in polyampholyte solutions were compared (Fig, 4), The hydrodynamic diameter observed by DLS in the polyampholyte solution of different $\mathrm{pH}$ was with around $50 \mathrm{~nm}$ nearly constant over the investigated $\mathrm{pH}$ area and quite similar to the dominant, lateral length scale of around $60 \mathrm{~nm}$ found in the adsorbed layers. A value comparable to the length scale of $179 \mathrm{~nm}$ at the $\mathrm{pH}_{\mathrm{IEP}}$ was not observed by DLS, because of the occurrence of the polyampholytic precipitation around the $\mathrm{pH}$ IEP of the polyampholyte. 

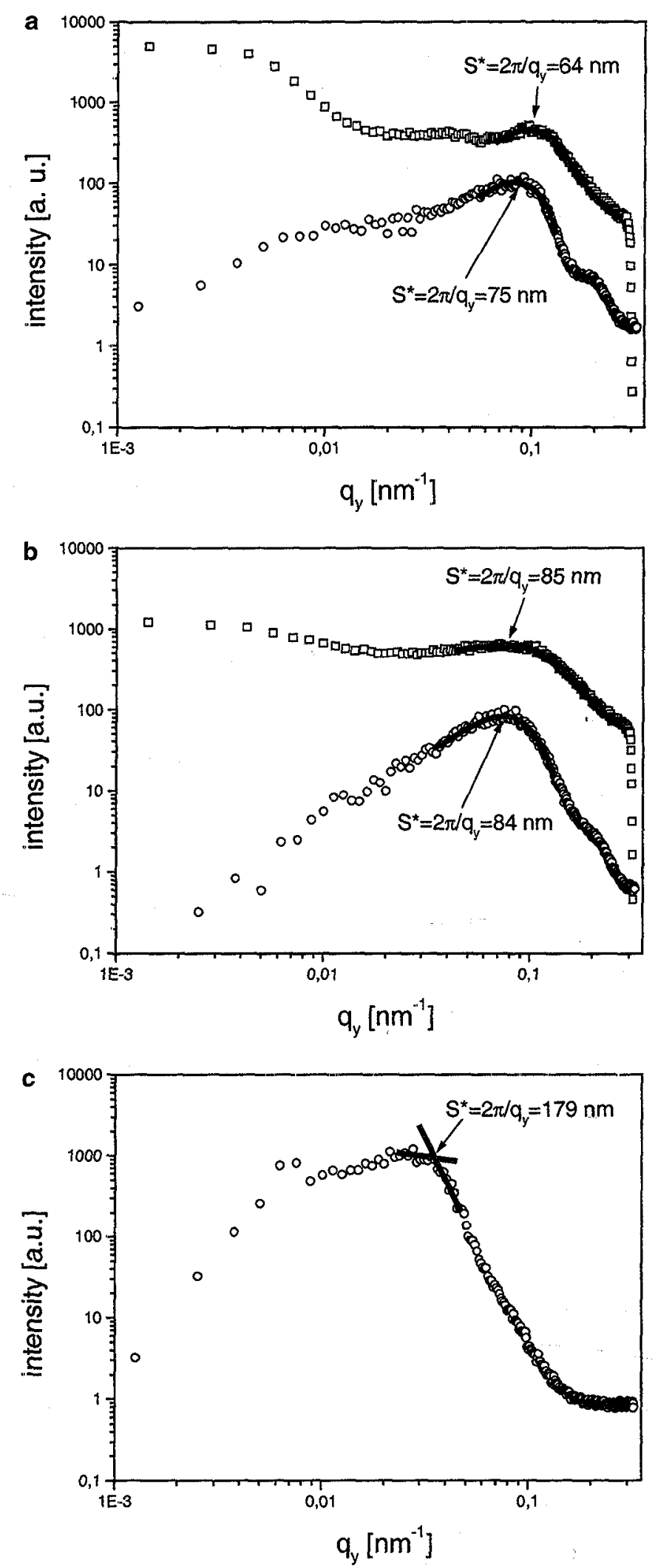

FIG. 3. PSD spectra $(O)$ and out of plane scans (D) of polyampholytic layers adsorbed at different $p H$ (a) $p H$ 6.1; (b) $p H$ 9.4; (c) $p H$ 8.5. The most prominent in-plane length scales are marked with an arrow. 


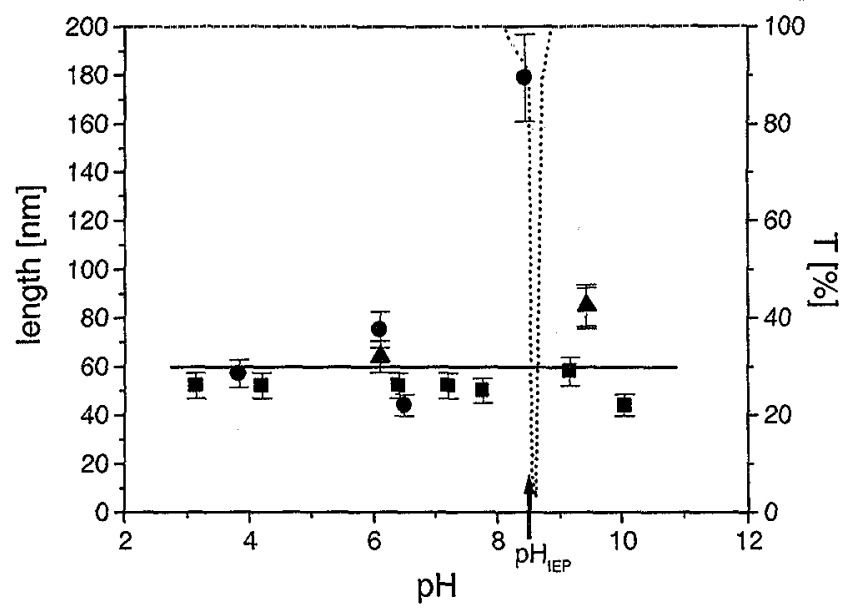

FIG.4. Hydrodynamic diameter of polyampholytic micelles in solution as function of $p H(\mathbf{\square})$. The dominant length scale from SFM topographies $(\bullet)$ and from GISAXS measurements $(\mathbf{\Lambda})$ of polyampholyte on silicon oxide prepared at different $\mathrm{pH}$. The solid line is a guide for the eye. The transmission T of a red laser light through the polyampholytic solution is shown as a dotted line as a function of $\mathrm{pH}$ The arrow below indicates the $\mathrm{pH}_{\text {IEP }} 8.5$ of the polyampholyte

The regular-sized polyampholytic structures in solution are explainable by micelle formation of the diblock polyampholyte [12-15]. Depending on the $\mathrm{pH}$ one of the polyampholyte blocks is more strongly charged than the other block and therefore mote hydrophilic. The other more hydrophobic block forms the core of the polyampholytic micelles. The formation of micelles from amphiphilic block copolymers in solution and their adsorption directly from solution are reported by many authors [15, 38] Also in the case of the investigated polyampholyte the adsorption of whole micelles explain the formation of similar-sized polyampholytic structures via adsorption at the silicon substrate A schematic drawing of the adsorption process of whole polyam-pholytic micelles is shown in Fig. 5. The charged surface of the micelle is electrostatically attracted by the oppositely charged substrate. This electrostatic attraction results in the adsorption of the polyampholyte Additionally the charged substrate should induce a polarization of the approaching micelle, so the attraction of the micelles to the surface is additionally increased $[39,40]$.

If the stability of the micelle is high during the adsorption process, only a small part of the micelle surface attracts to the substrate surface and acts as an anchor for the whole micelle (Fig. 5). The other polymer chains forming the adsorbed micelle are only connected via the hydrophobic core of the micelle to the silicon surface. Because of the electrostatic attraction between the anchor block of the polyampholyte and the substrate surface the distortion of the surface micelle during the drying: process affects only the height of the adsorbed structure and; not their lateral size. Therefore, during the drying of the sample the adsorbed micelle collapsed at the surface and a structure is obtained with a similar lateral diameter as that found in solution and at a decreased height [41].
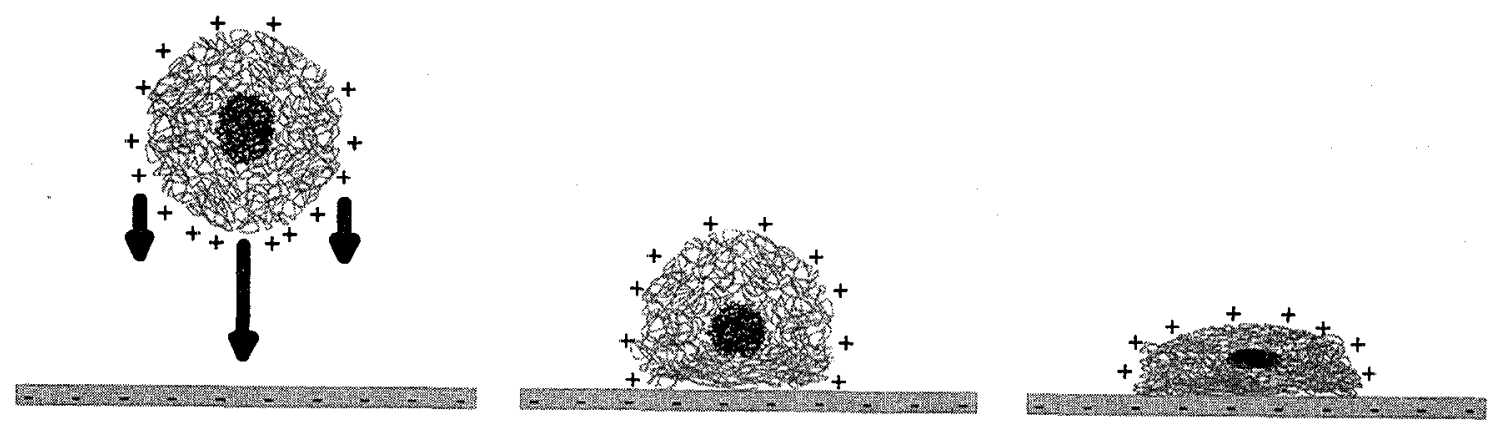

FIG. 5, Schematic drawing of the adsorption process of complete, positively charged polyampholytic micelles on top of the negatively charged substrate surface. The micelle basically collapses perpendicular to the substrate surface. 
Altogether the experiments suggest only a small amount of positively charged anchor blocks is directly attracted to the oppositely charged substrate.. The other polyampholytic chains placed in the micelle are not in direct contact with the substrate.. These chains should only be attracted via the hydrophobic core of the micelle. This schematic picture allows us to explain the highly adsorbed amount of more than $10 \mathrm{mg} / \mathrm{m}^{2}$, because the adsorbed layer is not only formed by directly attracted polyampholytic chains.
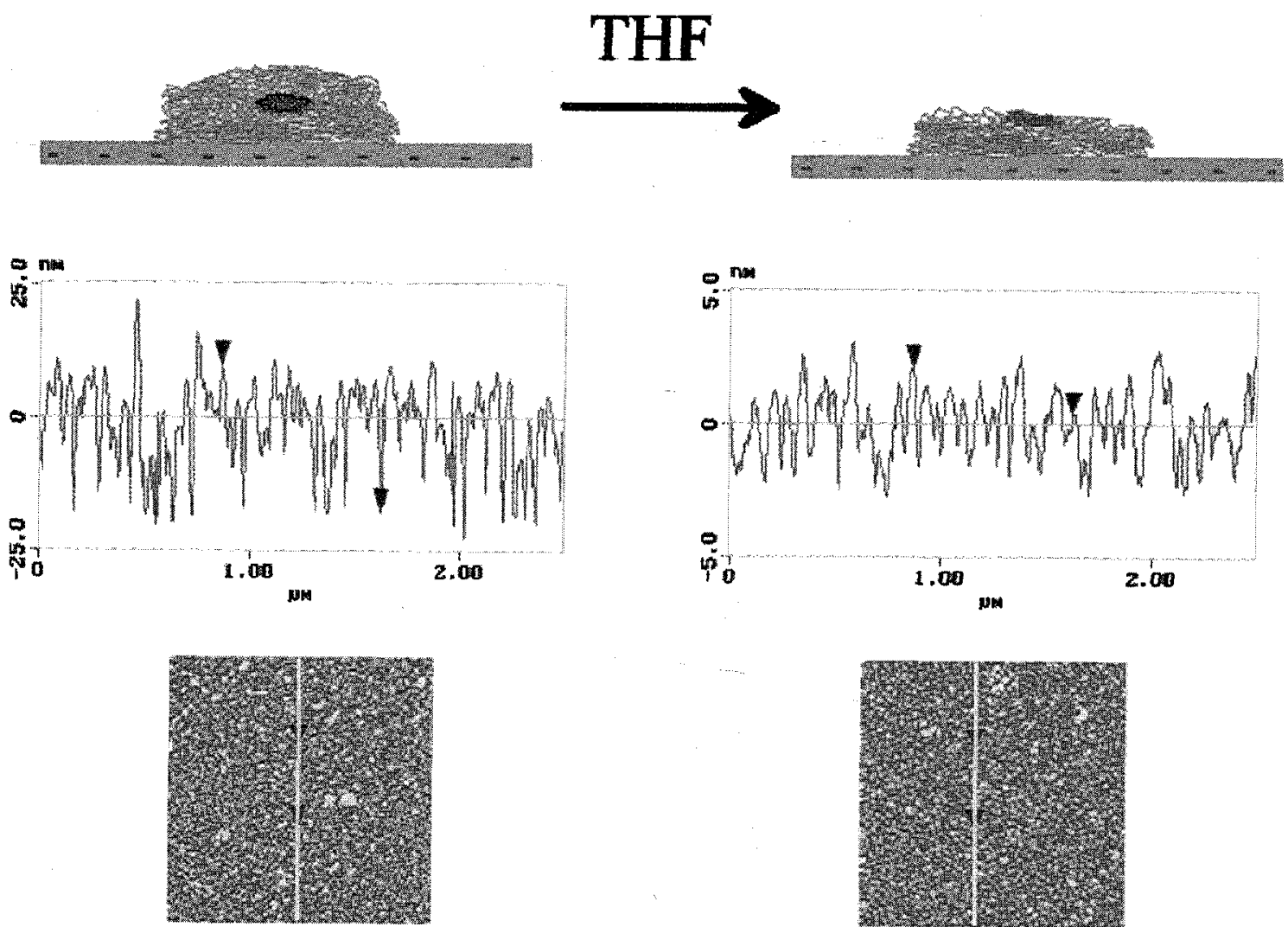

FIG. 6. Comparison of the adsorbed polyampholyte layer before (left) and after (right) treatment with THF. From top to bottom the schematic drawing, a line scan, and a topography of the layer observed by SFM are presented

Additionally the other part of the adsorbed micelle gives a contribution to the adsorbed amount. This explanation of the adsorbed micelle structure is supported by another experiment. If an adsorbed polyampholyte layer with $A=15.6 \mathrm{mg} / \mathrm{m}^{2}$ is treated with THF during a des-orption experiment, at the end only a small adsorbed amount of $A=2.2 \mathrm{mg} / \mathrm{m}^{2}$ is left. While the prominent lateral length of around $50 \mathrm{~nm}$ is less influenced by the desorption, the rms toughness of the polyampholyte layer decreased from 10.0 to $1.3 \mathrm{~nm}$ (Fig. 6). So the lateral sizes are nearly constant but their height decreased drastically over more than $80 \%$. THF as organic solvent is expected to solve the hydrophobic interactions inside the core of the adsorbed micelle. 


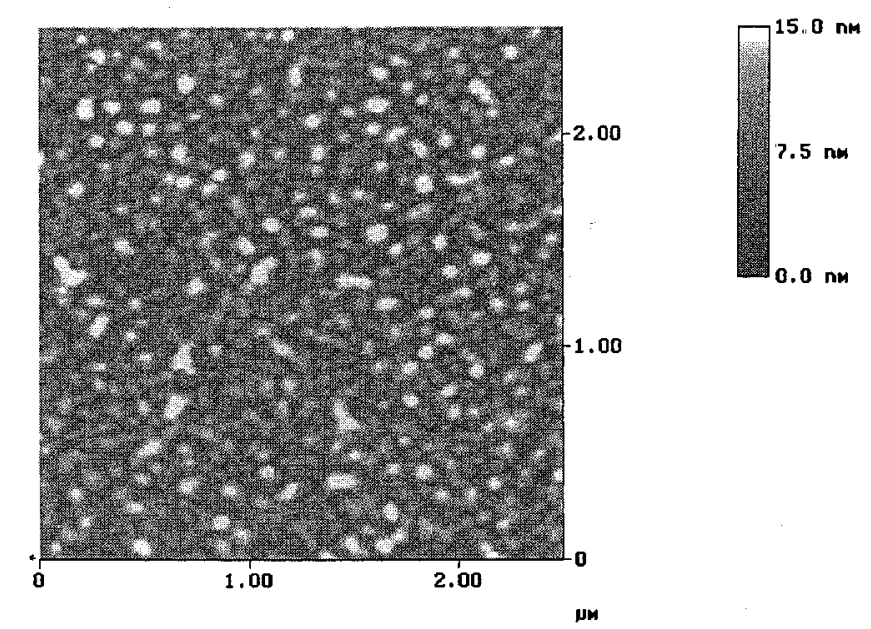

FIG. 7. SFM topography of adsorbed polyampholyte after annealing at $120^{\circ} \mathrm{C}$.

So the part of the micelle, which is attracted to the surface via the hydrophobic core, was desorbed and a decrease in the adsorbed amount appeared. The rest of adsorbed polyampholyte is strongly connected to the substrate via electrostatic interactions and is not desorbable by any organic solvent. While the top of the adsorbed micelles desorbs, the ground part shows no change in lateral structure and should be described as the stump of the micelle, which stays at the ground after the top is removed.

The stability of the regular adsorbed structures was also investigated as a function of temperature Until an annealing temperature of $80^{\circ} \mathrm{C}$ no significant changes in the surface topography was detectable using SFM. In contrast to this, an increase of up to $120^{\circ} \mathrm{C}$ lead to a drastic change in the surface topography. The regular adsorbed structures grew together and formed lateral larger structures of less regularity, while the height of the structures decreased (Fig. 7) This agglomeration behavior at higher temperatures is explained by the melting of the adsorbed micelles leading to an increased mobility of these structures

\section{CONCLUSIONS}

The adsorption of the diblock polyampholyte PMAA- $b$-PDMAEMA was investigated using ellipsometry, SFM, and GISAXS. Also the solution behavior of this polymer was investigated by DLS, This polyampholytic system was found to adsorb in highly regular-sized structures, in which lateral sizes were nearly independent from the $\mathrm{pH}$ of the adsorption solution. Thus we presented a method to create nearly monodisperse surface structures via polyampholyte adsorption, which might have important implications in the field of surface patterning Until now mostly uncharged molecules were under investigation.

Only directly at the IEP of the polyampholyte were larger adsorbed structures observed The regularity of the adsorbed structures were explained by the adsorption of whole micelles with similar diameters observed by DLS in solution During the adsorption process the shape of the micelles is expected to be stable, so only a small amount of the diblock chains forming the micelle is directly attracted to the substrate surface. The other polyampholyte chains in the adsorbed micelle are only connected to the surface via the hydrophobic core of the micelle The hydrophobically attached part is easily removable by treatment of the adsorbed layer with an organic solvent like THF In contrast to this, the electrostatically attracted part is not affected by THF treatment and stays at the substrate as a kind of micelle stump at the ground after the top is removed.

\section{ACKNOWLEDGMENTS}

We thank B. Silier and B, Müller (MPI-P, Mainz, Germany) for their help with dynamic light scattering measurements. For electrokinetic measurements we owe many thanks to A Manicke and $\mathrm{Dr}$ C Bellmann (IPF-Dresden, Germany), For their help at the USAX beamline BW4 at HASYLAB/DESY (Hamburg, Germany) we thank Dr.. G v. Kiosigk and S Cunis. We owe many thanks to the DFG Schwerpunkt Polyelektrolyte (Grant HC10-322 1009) for financial support. JFG and R.J are very much indebted to the Services Fédéraux des Affaires Scientifiques, Techniques et Culturelles for financial support in the frame of Poles d' Attraction Interuniversitaires: PAI 4-11: Chimie Supramole-culaire et Catalyse Supramoleculaire. JFG acknowledges the European Science Foundation program 


\section{SUPERNET,}

\section{REFERENCES}

[1]. Kudaibergenov, S. B, Adv. Polym Sci. 144,115 (1999). , [2 ] Dautzenberg, H..,, Jaeger, W, Kötz, J, Philipp, B, Seidel, C, and Stscherbina, D., "Polyelectrolytes." Hanser, Munich, 1994.

[3]. Watanabe, Y, Kubo, K,, and Sato, S,, Langmuir 15,4157 (1999).

[4]. Böhm, N , and Kulicke, W.-M., Colloid Polym Sci. 275, 73 (1997)

[5]. Janex, M. L., Chaplain, V., Counord, J L,, and Audebert, R, Colloid Polym Set. 275, 352 (1997).

[6] Förster, S, and Schmidt, M, Adv Polym. Sci, 120, 1.33 (1995).

[7] Blaakmeer, J., Böhmer, M. R.., Cohen Stuart, M A., and Fleer, G. J., Maao-molecules 23, 2301 (1990)

[8]. Styrkas,D,, A ,Bütün,Y,Lu,I.R.,Keddie,J.L.,andArmes,S.P.,Langmuir $\quad$ 16,5980 (2000).

[9] Sukhishvili, S. A., and Granick, S ,./ Chem Phys.. 109, 6861 (1998)

[10] Dobrynin, A. V, Rubinstein, M., and Joanny, J.-F.., Macromolecules 30, 4332 (1997)

[11]. Higgs, $P$ G , and Joanny, J.-F, J Chem Phys.. 94, 1543 (1991).

[12]. Gohy, I.-F, Creutz, S., Garcia, M, Mahltig, B.., Stamm, M, and Jérôme, I R,, Macromolecules $3.3,6378(2000)$.

[13], Goloub, T., de Keizei, A., and Cohen Stuart, M. A, Macromolecules 32, I 8441 (1999) $--4$

[14], Walter, H, Müller-Buschbaum, R, Gutmann, J. S, Lorenz-Haas, C, Harrats, G, Jérôme, R., and Stamm, M., Langmuir 15, 6984 (1999).

[15]. Mahltig, B,,, Gohy, T -F, Jérôme, R., Bellmann, C , and Stamm, M,„, Colloid Polym Sci 278, 502 (2000).

[16], Kamiyama, Y, and Israelachvilli, J, Macromolecules 25, 5081 (1992)

[17] Netz, R. R., and Joanny, .J.-F, Macromolecules 31, 5123 (1998)

[18] Neyret, S, Ouali, L., Candau, F.., and Pefferkorn, E,../.. Colloid Interface Sci 176, 86 (1995).

[19]. Blaakmeer, J,., Cohen Stuart, M. A., and Fleer, G J,, J Colloid Interface Sci. 140,314(1990).

[20] Mahltig, B , Walter, H., Harrats, C., Müller-Buschbaum, P, Jérôme, R, and .Stamm, M , Phys Chem Chem. Phys.. 1, 3853 (1999)

[21]. Walter, H , Harrats, C., Müller-Buschbaum, P, Jérôme, R., and Stamm, M , Langmuir 15, 1260 (1999)

[22] Mahltig, B., Gohy, J.-R., Jérôme, R., and Stamm, M., /. Polym Sci B Polym Phys. 39,709(2001).

[23]. Creutz, S, Teyssié, P, and Jérôme, R, Macromolecules 30, 6 (1997)

[24] Creutz, S..; van Stam, J.., Antoun, S , De Schryer, F. C, and Jerome, R., Maaomolecules 30, 4078 (1997)

[25]. Antoun, S.., Teyssié, P, and Jérôme, R, Macromolecules 30, 1556 (1997)..

[26] Harke, M.., Teppner, R., Schulz, O , Motschmann, H., and Orendi, H.., Rev Sci lnstrum 68,3130(1997). [27]. Azzam, R. M. A, and Bashara, N M., "Ellipsometiy and Polarized Light" North-Holland, Amsterdam, 1979

[28] Mes, E P C , Kok, W T, Poppe, H and Tijssen, R, / Polym. Sci B Polym Phys 37,593 (1999).

[29] Dumas, P., Bouffakhreddine, B , Amra, C, Vatel, O.., Andre, E., Gaindo, R, and Salvan,

F.,Europhys Lett, 22, 717 (1993)

[30] Gutmann, J S., Müller-Buschbaum, P., and Stamm, M , Faraday Discuss. 112,285(1999)

[31]. Gehrke, R., Rev Set lustrum 63, 455 (1992)

[32]. Müller-Buschbaum, P, Vanhoorne, P., Scheumann, V, and Stamm, M., Ewophys Lett 40, 655 (1997).

[33] Müller-Buschbaum, P, Casagrande, M,„, Gutmann, J, Kuhlmann, I, Stamm, M., von Krosigk, G, Lode, U, Cunis, S., and Gehrke, R.., Eu-rophys Lett 42,517 (1998)

[34] Salditt, T, Metzger, T H., Peisl,.T , and Goerigk,G,..7 Phys DrAppl. Phys 28, 236 (1995)

[35] Patrickios, C. S., Sharma, L. R, Armes, S. P, and Billinigham, N. C, Langmuir 15, 1613 (1999).

[36] Shubin, V, and Linse, P.,... Phys Chem 99,1285 (1995).

[37]. v. d Steeg, H G M, Cohen Stuart, M A.., de Keizer, A., and Bijsterbosch, B H., Langmuir 8, 2538 (1992).

[38] Meiners, J. C, Quintel-Ritzi, A, Mlynek, J., Elbs, H., and Krausch, G., Macromolecules 30,4945 (1997)

[39] Joanny, J..-F..../. Phys. II France 4, 1281 (1994)

[40] Dobrynin, A, V, Obukhov, S. P, and Rubinstein, M., Macromolecules .32, 5689 (1999)

[41]. Spatz, J. P, Sheiko, S., and Möller, M, Macromolecules 29, 3220 (1996) 